\title{
The neural correlates of well-being: A systematic review of the human neuroimaging and neuropsychological literature
}

\author{
Marcie L. King ${ }^{1}$ (D) \\ Published online: 6 May 2019 \\ (C) The Psychonomic Society, Inc. 2019
}

\begin{abstract}
What it means to be well and to achieve well-being is fundamental to the human condition. Scholars of many disciplines have attempted to define well-being and to investigate the behavioral and neural correlates of well-being. Despite many decades of inquiry into well-being, much remains unknown. The study of well-being has evolved over time, shifting in focus and methodology. Many recent investigations into well-being have taken a neuroscientific approach to try to bolster understanding of this complex construct. A growing body of literature has directly examined the association between well-being and the brain. The current review synthesizes the extant literature regarding the neural correlates of trait-like well-being (i.e., the propensity to live according to one's true nature). Although reported associations between well-being and the brain varied, some notable patterns were evidenced in the literature. In particular, the strongest and most consistent association emerged between well-being and the anterior cingulate cortex. In addition, patterns of association between well-being and the orbitofrontal cortex, posterior cingulate cortex, superior temporal gyrus, and thalamus emerged. These regions largely comprise the salience and default mode networks, suggesting a possible relationship between well-being and brain networks involved in the integration of relevant and significant stimuli. Various methodological concerns are addressed and recommendations for future research are discussed.
\end{abstract}

Keywords Well-being $\cdot$ Neural correlates $\cdot$ Neuroimaging

\section{Introduction}

Well-being is a complex trait-like construct, referring to optimal functioning (Lyubomirsky, Sheldon, \& Schkade, 2005; Ryan \& Deci, 2001). For centuries, people have attempted to understand the essential features of well-being. Scholars dating as far back as the ancient Greeks have postulated and theorized about what it means to be well and how to achieve well-being. In fact, the question "what is well-being?" is arguably a central question of human self-awareness. As creatures with both the capacity to reflect on thoughts and actions and with the inclination to reduce broad concepts into small components that can be labeled, people have spent many years attempting to define well-being. However, given that well-

Electronic supplementary material The online version of this article (https://doi.org/10.3758/s13415-019-00720-4) contains supplementary material, which is available to authorized users.

Marcie L. King

Marcie-king@uiowa.edu

1 Department of Psychological and Brain Sciences, University of Iowa, W311 Seashore Hall, Iowa City, IA 52242, USA being can be understood in the context of many domains of life (e.g., health, economics, social relations, politics) and can be examined from many points of view (e.g., individual, societal), a parsimonious definition of well-being has yet to, and may never, emerge. While a simple definition of well-being may be elusive, rigorous examinations of well-being are critical to our understanding of the construct.

Despite a long-standing interest in well-being, the field of psychology has devoted much more time and attention to the investigation of human suffering and unhappiness than to the causes and consequences of positive functioning (Ryan \& Deci, 2001; Ryff, 1989). Generally, research focusing on the underpinnings of psychopathology has been most common in psychological literature. The past 60 years, however, have seen an increase in work examining well-being (Keyes, Shmotkin, \& Ryff, 2002; Ryan \& Deci, 2001; Seligman \& Csikszentmihalyi, 2014). This move toward investigating well-being may be reflective of a growing awareness that well-being is not simply the absence of mental illness.

The same asymmetry in the study of well-being evident in psychology exists in the field of neuroscience. Whereas thousands of studies have investigated the neural correlates of psychopathology, far fewer studies have investigated the 
neural correlates of well-being. Just as elucidating the neural correlates of mental illness has been critically important to understanding psychopathology and in the development of interventions for mental illness, so too will elucidating the neural correlates of well-being be critically important to understanding what it means to be well and to enhancing wellbeing. The purpose of the current review is to systematically investigate the relationship between well-being and the brain, reviewing the small but growing body of neuroscientific research examining the neural correlates of well-being.

\section{Investigating well-being}

Investigations of well-being have been largely guided by two streams of inquiry into the question of what it means to lead a "good life" (Keyes et al., 2002; Ryan \& Deci, 2001). Historically, a common framework for understanding wellbeing distinguishes between hedonic well-being (hedonia), which can be traced back to the Greek philosophers Aristippus (435-366 BCE) and Epicurus (342-270 BCE) (Peterson, Park, \& Seligman, 2005), and eudaimonic wellbeing (eudaimonia), which can be traced back to Aristotle (384-322 BCE) (Bradburn, 1969; Waterman, 1984; Ryff, 1989). From a hedonic well-being perspective, a good life (translated by many as simply "happiness") is the totality of a person's pleasurable moments (Ryan \& Deci, 2001). From a eudaimonic well-being perspective, a good life, or "happiness," is not a consequence of satisfying appetites and feeling good, but instead stems from "activity of the soul in accordance with perfect virtue" (Ross/Aristotle, 1925, p.11).

Both hedonic and eudaimonic traditions of well-being have perpetuated throughout history. Religious leaders, political thinkers, philosophers, and scientists alike have used these perspectives of well-being as foundations for further theorizing on what it means to live well and how to achieve wellbeing. The terms most frequently used in the contemporary investigation of well-being in the fields of psychology and neuroscience include subjective well-being and psychological well-being and are thought to stem from hedonic and eudaimonic traditions of well-being, respectively.

The construct of subjective well-being is operationalized as consisting of three separable components: positive affect, lack of negative affect, and satisfaction with life (Andrews \& Withey, 1976; Lucas, Diener, \& Suh, 1996). Although subjective wellbeing is framed as the "new hedonic psychology," this approach to well-being actually focuses on a much broader conceptualization of hedonism which includes preferences and pleasures of both the mind and the body and includes cognitive judgments about the good and bad elements of life (Disabato et al., 2016; Diener, Emmons, Larsen, \& Griffin, 1985; Kahneman, Diener, \& Schwarz, 1999; Ryan \& Deci, 2001).

Psychological well-being provides a theory-driven formulation of well-being, integrating aspects of the "good life" postulated by Aristotle with perspectives of positive psychological functioning, such as Maslow's (1968) conception of self-actualization, Rogers's (1961) understanding of the fully functioning person, Jung's (1933) theory of individuation, and Allport's (1961) theory of maturity. Psychological well-being is operationalized along six dimensions: self-acceptance, purpose in life, personal growth, positive relations with others, environmental mastery, and autonomy (Ryff, 1989).

While subjective well-being and psychological well-being stem from hedonic and eudaimonic traditions of well-being and often are studied independently of one another, over time they have become less distinct. Current models of well-being suggest that subjective well-being and psychological wellbeing are highly correlated and lack discriminant validity (Disabato et al., 2016; Keyes et al., 2002). Measures of wellbeing often are categorized by their author's as reflecting a hedonic or eudaimonic approach to the construct; however, rigidly differentiating between these constructs may not be meaningful and may further confuse an already complex concept. In a review conducted by Cooke, Melchert, \& Connor (2016), the authors examined various well-being measures falling within four categories of conceptualizations of wellbeing: hedonic well-being, eudaimonic well-being, quality of life, and wellness. The authors identified 42 well-being measurement instruments, capturing 30 dimensions of positive functioning. Broadly, measures tapped into psychological, psychobiological (e.g., health and physical), and psychosocial well-being. The authors found that measures varied significantly in terms of their explicit operationalization of the constructs they were trying to assess. Although some dimensions of well-being, such as life satisfaction, positive affect, and positive relations with others, were more commonly tapped into than others, no single domain was identified in more than $50 \%$ of the instruments. Noting the high degrees of divergence in measurement instruments both between and within theoretical categories (e.g., hedonic, eudaimonic), Cooke et al. (2016) concluded that " “...there is little or no consensus as to what constitutes well-being and how it should be measured" (p. 748). This supports the stance that, at this stage of investigation, it may be more meaningful to investigate well-being as a single overarching construct rather than attempting to reduce it into component parts. Defining wellbeing as "optimal psychological functioning," operationalized according to both subjective and psychological conceptions of well-being, may be a more useful approach to the scientific study of well-being, and is the approach taken in the current review.

\section{Neuroscience of well-being}

A small body of work has directly examined the neural correlates of well-being. However, to date, a comprehensive examination of the extant literature on the neural correlates of well- 
being has not been conducted. One previous study reviewing the neural correlates of well-being suggested that gray matter volume in the insula and the precuneus may be positively associated with subjective and psychological well-being, respectively (Machado \& Cantilino, 2017). This brief review only included two studies, and the discussion of the potential relationship between well-being and the brain was necessarily speculative.

Additional empirical work points to associations between well-being and a wide range of brain regions, spanning nearly the entire cortex. This may in part reflect the fact that neuroimaging research has evolved quickly over the past two decades, resulting in an enormous literature. The high degree of variability in this literature, combined with the large number of neuroimaging studies published, makes it increasingly difficult to distinguish spurious from replicable findings (Müller et al., 2017). In addition, various instruments have been used to measure well-being in neuroscientific investigations, adding further variability to the literature. Taking care to address differences in methodologies across studies, and the associated implications of such differences, an overview of the state of the neuroscience of well-being may help to identify whether there are any brain areas that are consistently associated with well-being. Synthesizing the extant neuroscientific literature on well-being will be critical to the field moving forward. The current systematic narrative review offers a comprehensive analysis of the neuroscientific literature on wellbeing, contributing to our current understanding of the neural correlates of well-being.

\section{Methods}

\section{Search strategy and outcome}

Studies for the current review were identified in September 2017 and updated in June 2018 through searches conducted in PubMed, PsycInfo, and Embase databases. An iterative process of gathering and evaluating terms was undertaken to maximize search sensitivity and specificity. Comprehensive strategies, including subject methods (which use prescribed database-specific concept indexes to capture relevant records), keyword methods (which use specific words to capture relevant records), and customized filters were used in the search. Best practices for comprehensive literature reviews dictate the use of both subject and keyword methods (Higgins \& Green, 2011). No timeframe exclusions based on publication date were used. Detailed search strategies for all databases are available upon request.

The literature search produced 1468 unique studies. The large number of studies produced by the search reflects a broad sampling of the literature. To narrow the search, article titles and abstracts of the 1,468 studies were screened for potential inclusion. This initial screening process yielded 142 potential articles for inclusion. Full-text versions of relevant articles were then obtained and assessed for eligibility. Of the 142 studies assessed, 22 studies were included in the review (Fig. 1).

\section{Eligibility criteria}

To meet inclusion criteria, studies were required to include: adult populations (aged 18-100 years), at least one measure of neural correlates, and at least one measure of well-being. With the exception of neuropsychological studies examining patients with stable, focal brain lesions, included studies were limited to normal healthy adult populations. Included measures of neural correlates consisted of MRI (structural), functional (f)MRI (resting-state regional activity and functional connectivity), PET, EEG (at baseline or rest), and brain scans of lesions. Included measures of well-being consisted of selfreport measures that have been shown to be both reliable and valid across various populations (see Appendix 1 for a list of included measures and related references for reliability and validity information for each measure). Because well-being was defined for the purpose of this review as "optimal psychological functioning" and was operationalized to include components of both subjective and psychological well-being, studies using measures of either or both subjective and psychological well-being were included in the current review. All included measures can be used to assess well-being as a trait (rather than a state). No mood or emotion induction paradigms were included in the current review. Only peer-reviewed studies published in English were included in the current review. Studies that did not meet these criteria were excluded (see Appendix 2 for detailed exclusion information).

General information regarding the studies included in the current review is first provided in the results below. Following this overview, detailed findings from each study are organized according to gross anatomical brain structure (frontal lobe, parietal lobe, temporal lobe, occipital lobe, subcortical areas, other).

\section{Results}

The 22 studies included in the current review provide a broad snapshot of the state of the literature examining the association between well-being and the brain. While each study included shared a foundational aim to elucidate the relationship between well-being and the brain, the methods and techniques by which this relationship was investigated proved heterogeneous across the literature.

Studies varied both in terms of neuroimaging methodologies and in terms of instruments used to measure well-being. Seven studies used structural MRI techniques (Fritz et al., 


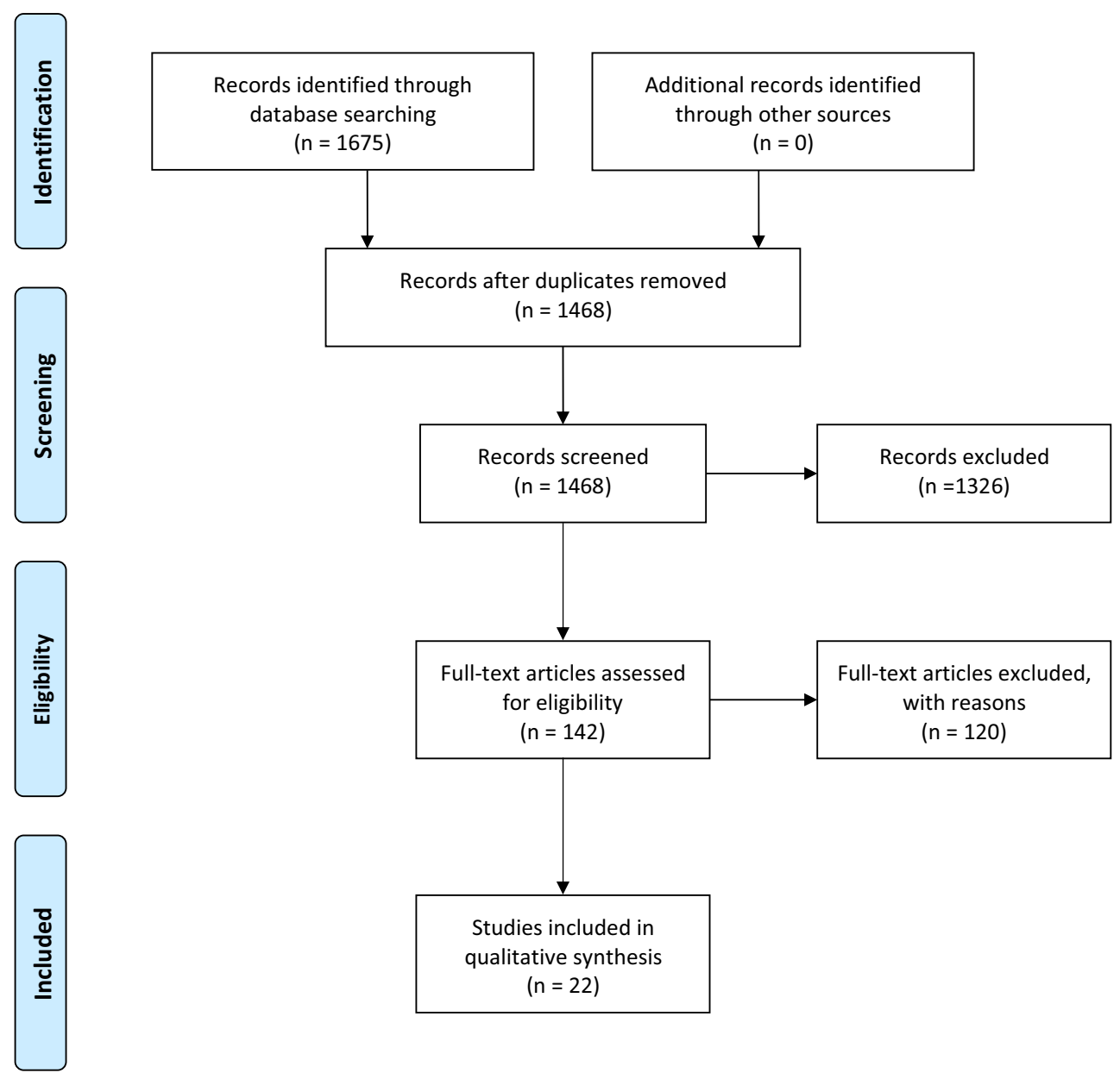

Fig. 1. Flow diagram of included studies

2016; Kong, Ding, et al., 2015; Kong, Hu, Xue, Song, \& Liu, 2015; Lewis, Kanai, Rees, \& Bates, 2014; Matsunaga et al., 2016; Sato et al., 2015; Takeuchi et al., 2014), nine studies used fMRI techniques (Hermes, Hagemann, Naumann, \& Walter, 2011; Kong, Hu, Wang, Song, \& Liu, 2015; Kong, Liu, et al., 2015; Kong, Wang, Hu, \& Liu, 2015; Kong, Wang, Song, \& Liu, 2016; Kong, Xue, \& Wang, 2016; Luo et al., 2014; Luo, Kong, Qi, You, \& Huang, 2016; Waytz, Hershfield, \& Tamir, 2015), two studies used PET imaging techniques (Martikainen et al., 2015; Volkow et al., 2011), and four studies used EEG (De Pascalis, Cozzuto, Caprara, \& Alessandri, 2013; Hagemann et al., 1999; Tomarken, Davidson, Wheeler, \& Doss, 1992; Urry et al., 2004). No studies examining the relationship between well-being and brain lesion location were identified as meeting criteria for the current review.

No two studies included in the current review used identical processing and analytical methods. Even within a given imaging modality (e.g., fMRI), different techniques were used. For example, of the nine studies included that used fMRI techniques, three used regional homogeneity, three used fractional amplitude of low frequency, one used arterial spin labelling, one used independent components analysis, and one reported use of a "functional connectivity analysis" to measure intrinsic brain activity. There are no standard procedures for processing images or neuroimaging results. Therefore, researchers can choose from a wide variety of acceptable and defensible methods. Differences in analysis type (e.g. wholebrain vs. region of interest), use of global signal regression, transformation of images into a common template space, and applied corrections for multiple comparisons were also apparent in every study. Although methodological techniques varied across studies, most techniques used were sufficiently justified. Authors of each study outlined their methodology in detail and reported what software packages and analysis choices were made (see Supplementary Material). For the purposes of the current review, all included studies were given equal weight in terms of their contribution to the results.

Multiple studies in the current review were identified as reporting on highly overlapping participant samples. Of the seven studies using structural MRI, five studies reported on independent cohorts of participants (Fritz et al., 2016; Lewis et al., 2014; Matsunaga et al., 2016; Sato et al., 2015; Takeuchi et al., 2014), and two studies reported on a highly 
overlapping sample of participants (Kong, Ding, et al., 2015; Kong, Hu, Xue, et al., 2015). The nine studies examining the relationship between well-being and neural functioning using resting-state fMRI methods were comprised of four independent samples. In particular, Waytz et al. (2015) and Hermes et al. (2011) reported on independent cohorts of participants. Luo et al. (2016) expanded on results reported in Luo et al. (2014). Kong, Hu, Wang, et al. (2015), Kong, Liu, et al. (2015), Kong, Wang, Hu, et al. (2015), Kong, Wang, Song, et al. (2016), and Kong, Xue, et al. (2016) reported on highly overlapping cohorts of participants. The participant samples in the two studies using PET imaging techniques and in the four studies using EEG were all independent.

Fourteen measures of well-being were used in the studies included in the current review. The Positive Affect Negative Affect Schedule (PANAS; Watson, Clark, \& Tellegen, 1988) was used most frequently (10/22 studies), followed by the Scales of Psychological Well-Being (SPWB; Ryff, 1989) and the Satisfaction with Life Scales (SWLS; Diener, Emmons, Larsen, \& Griffin, 1985). The SPWB and SWLS were each used in five studies.

Where appropriate some brain areas were collapsed to simplify results. Specifically, the reported results regarding the anterior cingulate cortex (ACC) also consist of results reported in the rostral ACC, dorsal ACC, and anterior middle cingulate gyrus. In addition, results regarding the posterior cingulate cortex (PCC) also consist of results reported in the posterior middle cingulate gyrus. Lastly, the reported results in the superior temporal gyrus (STG) also consist of results reported in the posterior STG, Heschel's gyrus, Rolandic operculum, and planum temporale. All other brain regions were reported in the current review as named in the primary literature.

Table 1 provides information about sample characteristics, imaging methods, and well-being measures for each study. The following results summarize findings reported in the literature in terms of the absolute values of the associations. Further detail regarding direction of association and nonsignificant associations is provided in Table 2.

\section{Association between well-being and the frontal lobe}

Thirteen studies reported significant associations between well-being and various areas of the frontal lobe (Hermes et al., 2011; Kong, Ding, et al., 2015; Kong, Hu, Wang, et al., 2015; Kong, Hu, Xue, et al., 2015; Kong, Wang, et al., 2015; Kong, Wang, et al., 2016; Kong, Xue, et al., 2016; Luo et al., 2014; Luo et al., 2016; Matsunaga et al., 2016; Takeuchi et al., 2014; Urry et al., 2004; Volkow et al., 2011). Converging evidence supporting an association between well-being and the frontal lobe emerged across all imaging modalities.

Overall, associations were reported between well-being and both lateral and medial aspects of the frontal lobe. Using
EEG, Urry et al. (2004) found significant associations between well-being and fronto-central asymmetry, with higher levels of well-being associated with greater activity in the left frontal lobe at baseline. Similarly, using PET techniques, Volkow et al. (2011) reported widespread significant correlations between well-being and baseline glucose metabolism in both lateral and medial aspects of the frontal lobe in the right and left hemispheres (BA6, BA8, BA9, BA10). In two fMRI studies, Luo et al. $(2014,2016)$ reported a significant association between well-being and resting-state regional activity in the left and right medial PFC.

More spatially specific findings regarding the association between well-being and the frontal lobe were reported in various structural and functional MRI studies as well. On the lateral aspect of the PFC, significant associations were reported between well-being and the left rostrolateral PFC (Takeuchi et al., 2014), left dorsolateral PFC (Kong, Ding et al., 2015; Luo et al., 2014), right ventrolateral PFC (Luo et al., 2014), left and right superior frontal gyrus (Kong, Hu, Wang, et al., 2015; Luo et al., 2014), and left and right inferior frontal gyrus (Kong, Wang, et al., 2016; Hermes et al., 2011) (Fig. 2a). On the ventral and medial aspects of the PFC, significant associations were reported between well-being and the left and right orbitofrontal cortex (OFC) (Hermes et al., 2011; Kong, Hu, Wang, et al., 2015; Kong, Wang, et al., 2016; Volkow et al., 2011), left ventromedial PFC (Kong, Ding, et al., 2015), and the left and right anterior cingulate cortex (ACC) (Matsunaga et al., 2016; Takeuchi et al., 2014; Kong, Xue, et al., 2016; Hermes et al., 2011; Kong, Wang, et al., 2015; Luo et al., 2014; Volkow et al., 2011) (Fig. 2b). As discussed further below, the ACC was the most consistently reported area associated with well-being.

\section{Association between well-being and the parietal lobe}

Seven studies reported significant associations between wellbeing and regions in the parietal lobe (Kong, Ding, et al., 2015; Kong, Hu, Wang, et al., 2015; Luo et al., 2014; Luo et al., 2016; Sato et al., 2015; Volkow et al., 2011; Waytz et al., 2015). Two studies reported associations between well-being and lateral aspects of the parietal lobe. In particular, significant associations were reported between well-being and left inferior parietal lobule (Luo et al., 2016) and the left postcentral gyrus, located in the anterior lateral parietal lobe (Kong, Hu, Wang, et al., 2015) (Fig. 3a).

More consistent findings emerged between well-being and medial aspects of the parietal lobe (Fig. 3b). Four studies reported a significant association between well-being and the left and right posterior cingulate cortex (PCC; Volkow et al., 2011; Luo et al., 2014; Luo et al., 2016; Kong, Hu, Wang, et al., 2015). These findings converged across both fMRI and PET work. Located adjacent to the PCC, the left and right precuneus also were consistently shown to be associated with 
Table 1 Summary of methodological details for all studies

\begin{tabular}{|c|c|c|c|c|c|c|c|c|c|c|c|}
\hline \multirow[t]{2}{*}{ Study } & \multicolumn{4}{|c|}{ Sample characteristics } & \multicolumn{6}{|c|}{ Imaging methods } & \multirow{2}{*}{$\begin{array}{l}\text { Well-Being } \\
\text { measure }\end{array}$} \\
\hline & $\mathrm{N}$ & $\begin{array}{l}\text { Mean } \\
\text { age }\end{array}$ & $\begin{array}{l}\% \\
\text { Female }\end{array}$ & $\begin{array}{l}\% \\
\text { Right } \\
\text { handed }\end{array}$ & $\begin{array}{l}\text { Imaging } \\
\text { modality }\end{array}$ & $\begin{array}{l}\text { Analysis } \\
\text { technique }\end{array}$ & $\begin{array}{l}\text { Whole brain } \\
\text { coverage } \\
(\mathrm{Y} / \mathrm{N})\end{array}$ & $\begin{array}{l}\text { ROI } \\
\text { analyses } \\
\text { conducted } \\
(\mathrm{Y} / \mathrm{N})\end{array}$ & $\begin{array}{l}\text { Transformed } \\
\text { to common } \\
\text { template } \\
\text { (MNI/ } \\
\text { Talairach) }\end{array}$ & $\begin{array}{l}\text { Corrected } \\
\text { for multiple } \\
\text { comparisons } \\
(\mathrm{Y} / \mathrm{N})\end{array}$ & \\
\hline $\begin{array}{l}\text { De Pascalis } \\
\quad \text { et al., } \\
2013\end{array}$ & 51 & 24.1 & $100 \%$ & $100 \%$ & EEG & alpha power & $\begin{array}{l}32 \\
\text { electrodes }\end{array}$ & Y & MNI & $\mathrm{Y}$ & PANAS \\
\hline $\begin{array}{l}\text { Fritz et al., } \\
2016\end{array}$ & 29 & 48.7 & $69 \%$ & -- & $\begin{array}{l}\text { structural } \\
\text { MRI }\end{array}$ & $\begin{array}{l}\text { Gray matter } \\
\text { volume }\end{array}$ & $\mathrm{Y}$ & -- & MNI & $\mathrm{N}$ & SF-36 \\
\hline $\begin{array}{l}\text { Hagemann } \\
\text { et al., } \\
1999\end{array}$ & 36 & 23.1 & $67 \%$ & $100 \%$ & EEG & alpha power & $\begin{array}{l}10 \\
\text { electrodes }\end{array}$ & $\mathrm{Y}$ & -- & -- & PANAS \\
\hline $\begin{array}{l}\text { Hermes } \\
\text { et al., } \\
2011\end{array}$ & 38 & 24.5 & $50 \%$ & $100 \%$ & fMRI & ASL & $\mathrm{Y}$ & Y & MNI & $\begin{array}{l}\text { N (for } \\
\text { whole-- } \\
\text { brain } \\
\text { analyses); } \\
\text { Y (for ROI } \\
\text { analyses) }\end{array}$ & PANAS \\
\hline $\begin{array}{l}\text { Kong, Ding, } \\
\text { et al., } \\
2015\end{array}$ & 299 & 21.6 & $53 \%$ & $94 \%$ & $\begin{array}{l}\text { structural } \\
\text { MRI }\end{array}$ & VBM & $\mathrm{Y}$ & Y & MNI & $\mathrm{Y}$ & $\begin{array}{l}\text { SWLS, } \\
\text { PANAS, } \\
\text { RSES }\end{array}$ \\
\hline $\begin{array}{l}\text { Kong, Hu, } \\
\text { Wang, } \\
\text { et al., } \\
2015\end{array}$ & 294 & 21.56 & $54 \%$ & -- & fMRI & fALFF & $\mathrm{Y}$ & $\mathrm{N}$ & MNI & $\mathrm{Y}$ & $\begin{array}{l}\text { SWLS, } \\
\text { PANAS }\end{array}$ \\
\hline $\begin{array}{l}\text { Kong, Hu, } \\
\text { Xue, } \\
\text { et al., } \\
2015\end{array}$ & 294 & 21.57 & $53 \%$ & $94 \%$ & $\begin{array}{l}\text { structural } \\
\text { MRI }\end{array}$ & $\begin{array}{l}\text { Gray matter } \\
\text { volume }\end{array}$ & $\mathrm{Y}$ & -- & MNI & $\mathrm{Y}$ & SWBS \\
\hline $\begin{array}{l}\text { Kong, Liu, } \\
\text { et al., } \\
2015\end{array}$ & 286 & 21.55 & $54 \%$ & -- & fMRI & fALFF & $\mathrm{Y}$ & $\mathrm{N}$ & MNI & $\mathrm{Y}$ & SPWB \\
\hline $\begin{array}{l}\text { Kong, } \\
\text { Wang, } \\
\text { Hu, et al., } \\
2015\end{array}$ & 290 & 21.56 & $54 \%$ & $94 \%$ & fMRI & ReHo & $\mathrm{Y}$ & $\mathrm{N}$ & MNI & $\mathrm{Y}$ & SWLS \\
\hline $\begin{array}{l}\text { Kong, } \\
\text { Wang, } \\
\text { Song, } \\
\text { et al., } \\
2016\end{array}$ & 276 & 21.57 & $54 \%$ & -- & fMRI & ReHo & $\mathrm{Y}$ & $\mathrm{N}$ & MNI & $\mathrm{Y}$ & $\begin{array}{l}\text { SPWB, } \\
\text { PANAS }\end{array}$ \\
\hline $\begin{array}{l}\text { Kong, Xue, } \\
\text { et al., } \\
2016\end{array}$ & 292 & 21.56 & $54 \%$ & $94 \%$ & fMRI & fALFF & $\mathrm{Y}$ & $\mathrm{Y}$ & MNI & $\mathrm{Y}$ & SWBS, OHQ \\
\hline $\begin{array}{l}\text { Lewis et al., } \\
2014\end{array}$ & 70 & 24.6 & $60 \%$ & -- & $\begin{array}{l}\text { structural } \\
\text { MRI }\end{array}$ & VBM & $\mathrm{Y}$ & -- & MNI & $\mathrm{Y}$ & SPWB \\
\hline $\begin{array}{l}\text { Luo et al., } \\
2014\end{array}$ & 50 & 20.26 & $72 \%$ & -- & fMRI & ReHo & $\mathrm{Y}$ & $\mathrm{Y}$ & MNI & $\mathrm{Y}$ & $\begin{array}{l}\text { SHS, PANAS, } \\
\text { CHI }\end{array}$ \\
\hline $\begin{array}{l}\text { Luo et al., } \\
2016\end{array}$ & 148 & 20.9 & $55 \%$ & -- & fMRI & ICA & $\mathrm{Y}$ & $\mathrm{Y}$ & MNI & $\mathrm{Y}$ & $\mathrm{CHI}$ \\
\hline $\begin{array}{l}\text { Martikainen } \\
\text { et al., } \\
2015\end{array}$ & 16 & 35 & $44 \%$ & $100 \%$ & PET & $\begin{array}{l}\text { [11C]raclopride } \\
\text { (dopamine } \\
\text { binding) }\end{array}$ & Unclear & $\mathrm{Y}$ & MNI & $\mathrm{N}$ & PANAS \\
\hline $\begin{array}{l}\text { Matsunaga } \\
\text { et al., } \\
2016\end{array}$ & 106 & 21.4 & $54 \%$ & $100 \%$ & $\begin{array}{l}\text { structural } \\
\text { MRI }\end{array}$ & VBM & $\mathrm{Y}$ & -- & MNI & $\mathrm{Y}$ & SHS \\
\hline $\begin{array}{l}\text { Sato et al., } \\
2015\end{array}$ & 51 & 22.5 & $51 \%$ & $100 \%$ & $\begin{array}{l}\text { structural } \\
\text { MRI }\end{array}$ & VBM & $\mathrm{Y}$ & Y & MNI & Y & $\begin{array}{l}\text { SHS, PIL, } \\
\text { Emotional }\end{array}$ \\
\hline
\end{tabular}


Table 1 (continued)

\begin{tabular}{|c|c|c|c|c|c|c|c|c|c|c|c|}
\hline \multirow[t]{2}{*}{ Study } & \multicolumn{4}{|c|}{ Sample characteristics } & \multicolumn{6}{|c|}{ Imaging methods } & \multirow{2}{*}{$\begin{array}{l}\text { Well-Being } \\
\text { measure }\end{array}$} \\
\hline & $\mathrm{N}$ & $\begin{array}{l}\text { Mean } \\
\text { age }\end{array}$ & $\begin{array}{l}\% \\
\text { Female }\end{array}$ & $\begin{array}{l}\% \\
\text { Right } \\
\text { handed }\end{array}$ & $\begin{array}{l}\text { Imaging } \\
\text { modality }\end{array}$ & $\begin{array}{l}\text { Analysis } \\
\text { technique }\end{array}$ & $\begin{array}{l}\text { Whole brain } \\
\text { coverage } \\
(\mathrm{Y} / \mathrm{N})\end{array}$ & $\begin{array}{l}\text { ROI } \\
\text { analyses } \\
\text { conducted } \\
(\mathrm{Y} / \mathrm{N})\end{array}$ & $\begin{array}{l}\text { Transformed } \\
\text { to common } \\
\text { template } \\
\text { (MNI/ } \\
\text { Talairach) }\end{array}$ & $\begin{array}{l}\text { Corrected } \\
\text { for multiple } \\
\text { comparisons } \\
(\mathrm{Y} / \mathrm{N})\end{array}$ & \\
\hline & & & & & & & & & & & $\begin{array}{l}\text { Intensity } \\
\text { Scale }\end{array}$ \\
\hline $\begin{array}{c}\text { Takeuchi } \\
\text { et al., } \\
2014\end{array}$ & 159 & 21.4 & $43 \%$ & $100 \%$ & $\begin{array}{l}\text { structural } \\
\text { MRI }\end{array}$ & VBM & $\mathrm{Y}$ & -- & -- & $\mathrm{Y}$ & $\begin{array}{l}\text { Japanese } \\
\text { version of } \\
\text { WHOQO- } \\
\text { L-26 }\end{array}$ \\
\hline $\begin{array}{c}\text { Tomarken } \\
\text { et al., } \\
1992\end{array}$ & 90 & $\begin{array}{c}\text { range: } \\
17-- \\
21\end{array}$ & $100 \%$ & $100 \%$ & EEG & alpha power & $\begin{array}{l}14 \\
\text { electrodes }\end{array}$ & $\mathrm{Y}$ & -- & -- & PANAS \\
\hline $\begin{array}{l}\text { Urry et al., } \\
2004\end{array}$ & 84 & 58.49 & $49 \%$ & $100 \%$ & EEG & alpha power & $\begin{array}{l}29 \\
\text { electrodes }\end{array}$ & Y & -- & -- & $\begin{array}{c}\text { PANAS, } \\
\text { SWLS, } \\
\text { SPWB }\end{array}$ \\
\hline $\begin{array}{l}\text { Volkow } \\
\text { et al., } \\
2011\end{array}$ & 47 & 31.5 & $51 \%$ & -- & PET & $\begin{array}{l}\text { FDG-PET } \\
\text { (glucose } \\
\text { metabolism) }\end{array}$ & $\mathrm{Y}$ & $\mathrm{Y}$ & Talairach & $\mathrm{Y}$ & MPQ \\
\hline $\begin{array}{l}\text { Waytz et al., } \\
2015\end{array}$ & 84 & 25.34 & $51 \%$ & -- & fMRI & $\begin{array}{l}\text { Functional } \\
\text { connectivity } \\
\text { analysis }\end{array}$ & $\mathrm{Y}$ & $\mathrm{Y}$ & MNI & $\mathrm{N}$ & $\begin{array}{c}\text { MLQ, SWLS, } \\
\text { SPWB }\end{array}$ \\
\hline
\end{tabular}

PANAS = Positive Affect Negative Affect Schedule; SF-36 = The Short Form-36; SWLS = Satisfaction with Life Scales; RSES = Rosenberg Scales of Self-Esteem; SWBS = Social Well-Being Scale; SPWB = Scales of Psychological Well-Being; OHQ = Orientation to Happiness Questionnaire; SHS = Subjective Happiness Scale; CHI = Chinese Happiness Inventory; PIL = Purpose in Life Test; WHOQOL-26 = WHO Quality of Life-26; MPQ = Multidimensional Personality Questionnaire; MLQ = Meaning in Life Questionnaire

well-being, using both structural MRI and PET techniques (Sato et al., 2015; Volkow et al., 2011; Kong, Ding, et al., 2015). In line with the reported relationship between wellbeing and the precuneus, Waytz et al. (2015) conducted a resting-state network analysis and found a significant relationship between well-being and the resting-state functional connectivity of a network, including the retrosplenial cortex (which overlaps with the precuneus), parahippocampal cortex, and ventral inferior parietal lobule. While this analysis does not reveal the relationship between well-being and these regions individually, it provides additional evidence for an association between well-being and a network involving medial regions of the parietal and temporal lobes.

\section{Association between well-being and the temporal lobe}

Significant associations between well-being and various areas of the temporal lobe were reported in eight studies (Hermes et al., 2011; Kong, Ding, et al., 2015; Kong, Hu, Wang, et al., 2015; Kong, Liu, et al., 2015; Kong, Xue, et al., 2016; Luo et al., 2014; Tomarken et al., 1992; Volkow et al., 2011). Converging evidence emerged across structural MRI, fMRI, EEG, and PET studies. The majority of findings reported in the literature suggest an association between well-being and the superior temporal gyrus, extending medially. In particular, as shown in Fig. $4 \mathrm{a}$ and b, significant associations between well-being and the right and left superior temporal gyrus (Luo et al., 2014; Kong, Liu, et al., 2015; Kong, Xue, et al., 2016; Kong, Hu, Wang, et al., 2015; Hermes et al., 2011; Volkow et al., 2011), the right parahippocampal gyrus (Luo et al., 2014; Kong, Ding, et al., 2015), the left hippocampus (Luo et al., 2014), and the right amygdala (Kong, Hu, Wang, et al., 2015) were reported. Additional associations between well-being and the temporal lobe were reported in the left and right middle temporal gyrus (Volkow et al., 2011), the left inferior temporal gyrus (Kong, Hu, Wang, et al., 2015; Volkow et al., 2011), and the left temporal pole (Tomarken et al., 1992). Notably, PET imaging revealed a widespread association between well-being and the temporal lobe, including the lateral and medial aspects of the superior, middle, and inferior temporal gyri (BA20, BA21, BA22; Volkow et al., 2011).

\section{Association between well-being and the occipital lobe}

Two studies reported significant associations between wellbeing and the occipital lobe (Volkow et al., 2011; Kong, Hu, Wang, et al., 2015). Figure 5 illustrates these findings. Volkow et al. (2011) reported a significant correlation between well- 
Table 2. Summary of findings regarding the association between well-being and neural correlates

\begin{tabular}{|c|c|c|c|c|c|c|c|c|c|c|}
\hline & \multirow{2}{*}{$\begin{array}{l}\text { Frontal } \\
\text { lobe }\end{array}$} & \multirow{2}{*}{$\begin{array}{l}\text { Parietal } \\
\text { lobe }\end{array}$} & \multirow{2}{*}{$\begin{array}{l}\text { Temporal } \\
\text { lobe }\end{array}$} & \multirow{2}{*}{$\begin{array}{l}\text { Occipital } \\
\text { lobe }\end{array}$} & \multirow{2}{*}{$\begin{array}{l}\text { Subcortical } \\
\text { areas }\end{array}$} & \multicolumn{5}{|l|}{ Other } \\
\hline & & & & & & Insula & Brainstem & Cerebellum & $\begin{array}{l}\text { Whole } \\
\text { brain }\end{array}$ & $\begin{array}{l}\text { Central } \\
\text { asymmetry }\end{array}$ \\
\hline \multicolumn{11}{|c|}{ Summary of structural MRI findings } \\
\hline Fritz et al., 2016 & $\mathrm{nr}$ & $\mathrm{nr}$ & $\mathrm{nr}$ & $\mathrm{nr}$ & - & $\mathrm{nr}$ & - & $\mathrm{nr}$ & - & $\mathrm{nr}$ \\
\hline Kong, Ding, et al., 2015 & $-*$ & $-*$ & $+*$ & $\mathrm{nr}$ & $\mathrm{nr}$ & $\mathrm{nr}$ & $\mathrm{nr}$ & $\mathrm{nr}$ & $\mathrm{nr}$ & $\mathrm{nr}$ \\
\hline $\begin{array}{l}\text { Kong, Hu, Xue, et al., } \\
2015\end{array}$ & $-*$ & $\mathrm{nr}$ & $\mathrm{nr}$ & $\mathrm{nr}$ & $\mathrm{nr}$ & $\mathrm{nr}$ & $\mathrm{nr}$ & $\mathrm{nr}$ & $\mathrm{nr}$ & $\mathrm{nr}$ \\
\hline Lewis et al., 2014 & $\mathrm{nr}$ & $\mathrm{nr}$ & $\mathrm{nr}$ & $\mathrm{nr}$ & $\mathrm{nr}$ & $+*$ & $\mathrm{nr}$ & $\mathrm{nr}$ & $\mathrm{nr}$ & $\mathrm{nr}$ \\
\hline Matsunaga et al., 2016 & $+*$ & $\mathrm{nr}$ & $\mathrm{nr}$ & $\mathrm{nr}$ & $\mathrm{nr}$ & $\mathrm{nr}$ & $\mathrm{nr}$ & $\mathrm{nr}$ & $\mathrm{nr}$ & $\mathrm{nr}$ \\
\hline Sato et al., 2015 & - & $+*$ & $\mathrm{nr}$ & $\mathrm{nr}$ & $\mathrm{nr}$ & - & $\mathrm{nr}$ & - & $\mathrm{nr}$ & $\mathrm{nr}$ \\
\hline Takeuchi et al., 2014 & $-*$ & $\mathrm{nr}$ & $\mathrm{nr}$ & $\mathrm{nr}$ & $\mathrm{nr}$ & $\mathrm{nr}$ & $\mathrm{nr}$ & $\mathrm{nr}$ & $\mathrm{nr}$ & $\mathrm{nr}$ \\
\hline \multicolumn{11}{|c|}{ Summary of fMRI findings } \\
\hline Hermes et al., 2011 & $-*$ & $\mathrm{nr}$ & $-*$ & $\mathrm{nr}$ & $-*$ & $\mathrm{nr}$ & $\mathrm{nr}$ & $\mathrm{nr}$ & $\mathrm{nr}$ & $\mathrm{nr}$ \\
\hline $\begin{array}{l}\text { Kong, Hu, Wang et al., } \\
2015\end{array}$ & $-*$ & $+*$ & -*/+* & $\mathrm{nr}$ & $+*$ & $\mathrm{nr}$ & $\mathrm{nr}$ & $\mathrm{nr}$ & $\mathrm{nr}$ & $\mathrm{nr}$ \\
\hline Kong, Liu, et al., 2015 & $\mathrm{nr}$ & $\mathrm{nr}$ & $t^{*}$ & $\mathrm{nr}$ & $-* /+*$ & $\mathrm{nr}$ & $\mathrm{nr}$ & $\mathrm{nr}$ & $\mathrm{nr}$ & $\mathrm{nr}$ \\
\hline Kong, Wang, et al., 2015 & $-* / \mathrm{ns}$ & $\mathrm{nr}$ & $\mathrm{nr}$ & $\mathrm{nr}$ & $\mathrm{nr}$ & ns & $\mathrm{nr}$ & $\mathrm{nr}$ & $\mathrm{nr}$ & $\mathrm{nr}$ \\
\hline Kong, Wang, et al., 2016 & $-* /+*$ & $\mathrm{nr}$ & $\mathrm{nr}$ & $\mathrm{nr}$ & $\mathrm{nr}$ & ns & $\mathrm{nr}$ & $\mathrm{nr}$ & $\mathrm{nr}$ & $\mathrm{nr}$ \\
\hline Kong, Xue, et al., 2016 & $+* / \mathrm{ns}$ & $\mathrm{ns}$ & $t^{*}$ & $\mathrm{nr}$ & $t^{*}$ & $+*$ & $\mathrm{nr}$ & $\mathrm{nr}$ & $\mathrm{nr}$ & $\mathrm{nr}$ \\
\hline Luo et al., 2014 & $-* /+*$ & $+*$ & $t^{*}$ & $\mathrm{nr}$ & $-*$ & $\mathrm{nr}$ & $\mathrm{nr}$ & $\mathrm{nr}$ & $\mathrm{nr}$ & $\mathrm{nr}$ \\
\hline Luo et al., 2016 & $-*$ & $-*$ & $\mathrm{nr}$ & $\mathrm{nr}$ & $\mathrm{nr}$ & $\mathrm{nr}$ & $\mathrm{nr}$ & $\mathrm{nr}$ & $\mathrm{nr}$ & $\mathrm{nr}$ \\
\hline Waytz et al., 2015 & $\mathrm{nr}$ & $+*$ & $\mathrm{nr}$ & $\mathrm{nr}$ & $\mathrm{nr}$ & $\mathrm{nr}$ & $\mathrm{nr}$ & $\mathrm{nr}$ & $\mathrm{nr}$ & $\mathrm{nr}$ \\
\hline \multicolumn{11}{|l|}{ Summary of PET findings } \\
\hline Martikainen et al., 2015 & $\mathrm{nr}$ & $\mathrm{nr}$ & $\mathrm{nr}$ & $\mathrm{nr}$ & - & $\mathrm{nr}$ & $\mathrm{nr}$ & $\mathrm{nr}$ & $\mathrm{nr}$ & $\mathrm{nr}$ \\
\hline Volkow et al., 2011 & $+*$ & $+*$ & $+*$ & $+*$ & $\mathrm{nr}$ & $\mathrm{nr}$ & $\mathrm{nr}$ & $\mathrm{nr}$ & $+*$ & $\mathrm{nr}$ \\
\hline \multicolumn{11}{|l|}{ Summary of EEG findings } \\
\hline De Pascalis et al., 2013 & $-/+$ & - & $\mathrm{nr}$ & $\mathrm{nr}$ & $\mathrm{nr}$ & $\mathrm{nr}$ & $\mathrm{nr}$ & $\mathrm{nr}$ & $\mathrm{nr}$ & + \\
\hline Hagemann et al., 1999 & - & + & - & $\mathrm{nr}$ & $\mathrm{nr}$ & $\mathrm{nr}$ & $\mathrm{nr}$ & $\mathrm{nr}$ & $\mathrm{nr}$ & - \\
\hline Tomarken et al., 1992 & + & $\mathrm{nr}$ & $+*$ & $\mathrm{nr}$ & $\mathrm{nr}$ & $\mathrm{nr}$ & $\mathrm{nr}$ & $\mathrm{nr}$ & $\mathrm{nr}$ & $\mathrm{nr}$ \\
\hline Urry et al., 2004 & $+*$ & $\mathrm{nr}$ & $\mathrm{nr}$ & $\mathrm{nr}$ & $\mathrm{nr}$ & $\mathrm{nr}$ & $\mathrm{nr}$ & $\mathrm{nr}$ & $\mathrm{nr}$ & $\mathrm{nr}$ \\
\hline
\end{tabular}

$\mathrm{nr}=$ not reported

$\mathrm{ns}=$ reported as non-significant correlation (no direction of correlation provided)

+ positive correlation

- negative correlation

*Significant correlation

being and baseline glucose metabolism in the right and left fusiform. Similarly, Kong, Hu, Wang, et al. (2015) reported a significant association between well-being and resting-state regional activity in the right lingual gyrus.

\section{Association between well-being and the subcortical areas}

Five studies reported significant associations between wellbeing and resting-state regional activity in various subcortical structures (Hermes et al., 2011; Kong, Hu, Wang, et al., 2015; Kong, Liu, et al., 2015; Kong, Xue, et al., 2016; Luo et al., 2014). Significant associations were found between well- being and the left and right putamen (Luo et al., 2014; Hermes et al., 2011), left and right caudate (Hermes et al., 2011), and the left and right thalamus (Luo et al., 2014; Kong, $\mathrm{Hu}$, Wang, et al., 2015; Kong, Xue, et al., 2016; Kong, Liu, et al., 2015). In addition, Kong, Liu, et al. (2015) reported a significant correlation between well-being and resting-state functional connectivity between the thalamus and the insula.

\section{Association between well-being and other brain areas}

Insula Because the insula is not easily grouped with one of the major other lobes discussed, it was examined independently in 
a

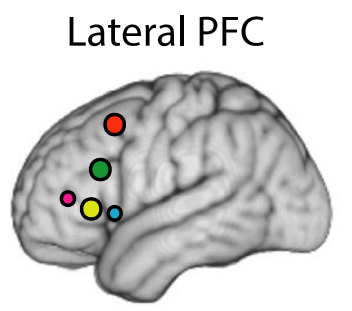

Number of studies:

- 000

b Ventral PFC

$1234+$

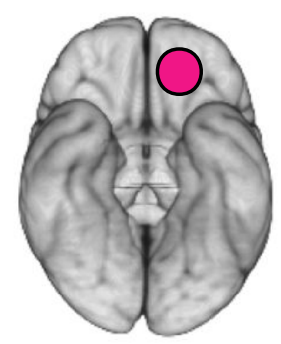

O OFC

- Ventromedial PFC

o ACC

\section{Medial PFC}

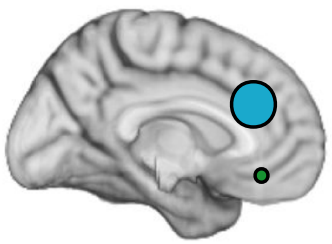

Fig. 2. Graphical illustration of reported findings in the frontal lobes. Circles are sized according to the number of studies reporting findings in the given location

a
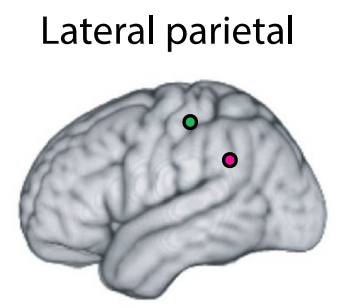

- Inferior parietal lobule

- Postcentral gyrus

\section{b}

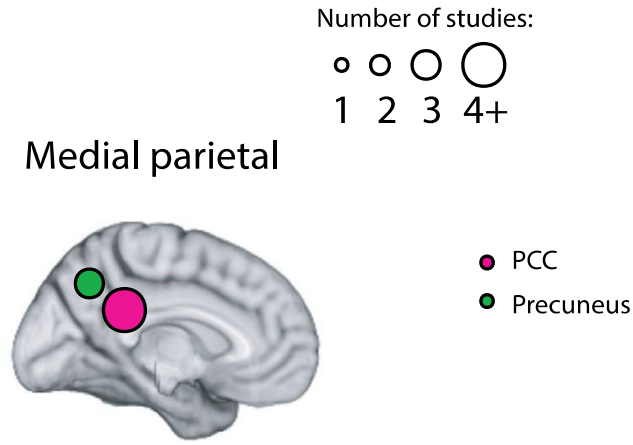

Fig. 3. Graphical illustration of reported findings in the parietal lobes. Circles are sized according to the number of studies reporting findings in the given location

a

\section{Lateral temporal}

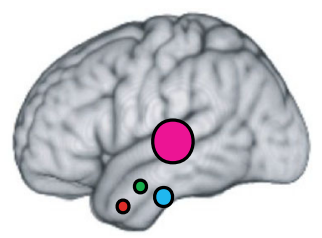

- Superior temporal gyrus

- Middle temporal gyrus

- Inferior temporal gyrus

- Temporal pole $\begin{array}{llll}\circ & 0 & \bigcirc & \bigcirc \\ 1 & 2 & 3 & 4+\end{array}$

b

\section{Medial temporal}

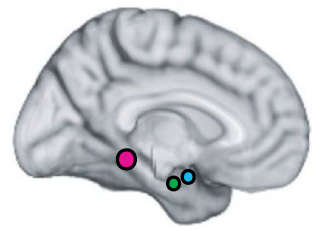

- Parahippocampal gyrus

- Hippocampus

- Amygdala

Fig. 4. Graphical illustration of reported findings in the temporal lobes. Circles are sized according to the number of studies reporting findings in the given location

the current review. Although few studies reported associations between well-being and the insula, some consistent results were evident in the literature. Lewis et al. (2014) found a significant correlation between well-being and gray matter volume in the right insula. In line with Lewis et al. (2014), Kong, Xue, et al. (2016) found a significant correlation between well-being and resting-state regional activity in the right insula.

Whole brain Volkow et al. (2011) reported a significant positive correlation between well-being and whole-brain baseline glucose metabolism. This finding suggests an overall association between well-being and the brain, but does not elucidate any specific brain area highly associated with well-being.

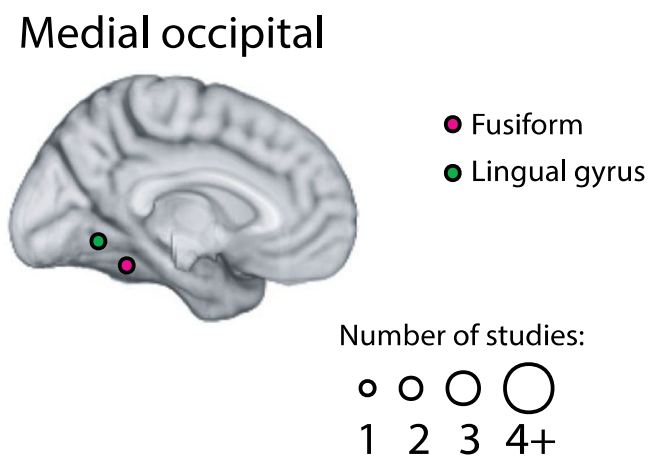

Fig. 5. Graphical illustration of reported findings in the occipital lobes. Circles are sized according to the number of studies reporting findings in the given location 


\section{Discussion}

The current review systematically investigated the relationship between well-being and the brain, moving toward a better understanding of this complex relationship. Sampling the literature broadly, this review revealed widespread reported associations between trait well-being, measured by self-report, and the brain. Patterns in the association between well-being and the brain emerged at varying levels of strength and consistency.

From a gross anatomical perspective, the relationship between well-being and the brain was most consistently reported in the frontal lobe followed by the temporal lobe, parietal lobe, subcortical areas, and occipital lobe. In particular, of the 22 studies included in the current review, 13 reported significant associations between well-being and frontal areas, 8 reported significant associations between well-being and temporal areas, 7 reported significant associations between well-being and parietal areas, 5 reported significant associations between well-being and subcortical areas, and 2 reported significant associations between well-being and occipital areas.

Overall, the most consistent and strongest association emerged between well-being and the ACC. With an estimated absolute effect size of $r=0.41$, a significant association between well-being and the ACC was reported in 7 of 22 studies, consisting of 6 independent samples. This association was evident across multiple imaging modalities, including structural MRI, fMRI, and PET.

In addition to the ACC, other patterns of association emerged between well-being and the OFC, PCC, STG, and thalamus. Average effect sizes for these associations ranged from $r=0.30$ (in the STG) to $r=0.40$ (in the PCC). These findings also varied in terms of consistency; four studies (consisting of 3 independent samples) reported significant associations between well-being and the OFC, four studies (consisting of 3 independent samples) reported significant associations between well-being and the PCC, six studies (consisting of 4 independent samples) reported significant associations between well-being and the STG, and four studies (consisting of 2 independent samples) reported significant associations between well-being and the thalamus.

Notably, there is not much basis in the literature reviewed here to make any strong predictions about the laterality of the association between well-being and the brain. Some work suggests that positive affective experience and positive emotional processing is related to left-sided frontal lobe activation, whereas negative affective experience and negative emotional processing is related to right-sided frontal activation (Davidson, 2004; Schaffer, Davidson, \& Saron, 1983). However, in the current review, relationships between well-being and the brain were reported in both the right and left hemispheres at a nearly equal frequency for most brain regions, leaving the question of the interaction between laterality and well-being open for further investigation.

\section{Interpretation and implications}

The intriguing patterns of association between well-being and the brain revealed in the current review warrant cautious interpretation and can be used to generate novel hypotheses regarding the neural correlates of well-being. Insights from the current review may not only be useful in fostering future theoretical and methodological advances in the investigation of the neural correlates of well-being but may also have important clinical implications.

The brain regions most consistently associated with wellbeing in the extant literature - the ACC, OFC, PCC, STG, and thalamus - are implicated in various high-level processes that, from a theoretical standpoint, likely play a role in well-being. The most consistent association between well-being and the brain emerged in the ACC. A large body of research suggests that the ACC is associated with many important cognitive and emotional functions, including conflict monitoring, error detection, motivation, emotion regulation, attention, and cognitive control (see Bush, Luu \& Posner, 2000 for review). These processes may be particularly important for optimal psychological functioning, or well-being. Past work shows that while experiencing positive events, focusing on the present moment and engaging in positive rumination promotes positive affect, a core component of well-being. In contrast, focusing on negative details of an experience dampens positive affect and negative rumination decreases life satisfaction (Quoidbach, Berry, Hansenne, \& Mikolajczak, 2010). In addition to the extensive functional repertoire of the ACC, the extensive neuroanatomical connections between the ACC and other cortical and subcortical regions (see Paus, 2001 for review) may allow for its unique involvement in well-being.

From a network perspective, it is notable that the ACC, OFC, PCC, STG, and thalamus largely comprise the salience network and the default mode network. The salience network consists of neural centers (e.g., ACC, OFC) involved in conflict monitoring, interoceptive-autonomic and reward processing and responds in a task-independent manner to cognitive, emotional, and homeostatic stimuli of personal salience (Peyron et al., 2000; Craig, 2002; Critchley, 2005). The default mode network consists of the ventromedial PFC (including the ventral ACC and OFC), PCC, and inferior lateral parietal lobe (for review see Buckner, Andrews-Hanna \& Schacter, 2008) and is activated during undirected, passive tasks, possibly reflecting neural activity associated with spontaneous internal thoughts (Andrews-Hanna, 2012). In a compelling review by Berridge and Kringelbach (2011), the authors examined the neural correlates of hedonic pleasure and theorized that the default mode network may somehow bridge hedonic and eudaimonic happiness, serving, at least in part, as a neural substrate of well-being.

One apparent point of functional overlap between the salience and default mode networks is that they are both 
implicated in the ability to integrate personally relevant and significant information (Seeley et al., 2007; Greicius, Krasnow, Reiss, \& Menon, 2003). A primary priority of the human nervous system is to maintain homeostasis in the context of a continuous inundation of internal and external inputs. The capacity to maintain equilibrium requires a system that can flexibly integrate highly processed external stimuli (e.g., sensory data from the environment) with complex internal stimuli (e.g., visceral and autonomic signals and internal thoughts) and use this information to determine what to do next. Well-being may in part reflect the brain's ability to integrate relevant and significant internal and extrapersonal stimuli in service of maintaining equilibrium.

The precise relationship between the salience and default mode networks is an area of open investigation. Research suggests that the default mode network may work in direct opposition to the salience network (Buckner et al., 2008; Seeley et al., 2007). This inverse relationship may result from the direct inhibition of the default mode network during activation of the salience network or may be maintained by a third region, such as the thalamus, which has been shown to play an important intermediary role in cortico-cortical interactions (Seeley et al., 2007; Greicius et al., 2003). Although further work is needed to elucidate the relationship between the salience and default mode networks systems, it is clear that these systems interact with and modulate one another (Buckner et al., 2008). A balanced interaction within and between these networks may be particularly relevant for well-being.

Perhaps not coincidentally, areas implicated in well-being in the current review have also been associated with major psychiatric illnesses, which often are in part defined by lack of well-being. In particular, aberrant functioning in the salience and default mode networks is linked to a wide range of mental illnesses, including depression and anxiety (Barrett \& Simmons, 2015; Hamilton, Farmer, Fogelman, \& Gotlib, 2015; Kaiser, Andrews-Hanna, Wager, \& Pizzagalli, 2015; Mulders, van Eijndhoven, Schene, Beckmann, \& Tendolkar, 2015). Extensive work has revealed a relationship between abnormal functioning in areas of the salience network (manifested as atypical autonomic interoceptive processing, such as heart rate variability, blood pressure) and depression (Barrett \& Simmons, 2015; Chen et al., 2017; Kemp et al., 2010). Similarly, a relationship between maladaptive rumination and hyperconnectivity in the default mode network has been reported (Hamilton et al., 2015; Kaiser et al., 2015; Mulders et al., 2015). Although well-being is not merely the absence of mental illness, these findings further support the hypothesis that well-being is, at least in part, related to typical functioning of the salience and default mode networks.

Findings from the current review thus tie together important pieces of largely disparate literatures which, taken together, have important possible therapeutic implications. Understanding the neural correlates of well-being and how they fit into 1) large-scale distributed functional networks and 2) current understandings of brain-behavior relationships is important both for promoting well-being and for developing more targeted treatments of mental illness. If, as hypothesized, well-being is associated with the ability to integrate personally relevant and significant information via the salience and default mode networks, cognitive behavioral therapies focusing on enhancing cognitive control of attention and using relevant and significant information to update behavior may be particularly important for promoting well-being. In addition, an emerging body of work investigating the anxiolytic and antidepressant properties of Floatation-Reduced Environmental Stimulation Therapy (Floatation-REST) suggests that increased interoceptive awareness and enhanced attention to autonomic processes, including breathing and heart rate, reduce anxiety in both clinically anxious and healthy populations (Feinstein, Khalsa, Yeh, Al Zoubi, et al., 2018; Feinstein, Khalsa, Yeh, Wohlrab, et al., 2018). Although nascent, this work fits with the idea that promoting interoceptive awareness, possibly through sensory deprivation, such as that induced in the Floatation-REST environment, can reduce anxiety and promote well-being. Lastly, to the extent that the salience and default mode networks are accessible by stimulation (e.g., transcranial magnetic stimulation) or medication, they may be relevant targets for biological treatments of mental illness.

\section{Limitations}

The current review revealed intriguing patterns of association between well-being and the brain; however, due to various limitations, should be interpreted cautiously. Differences in measurement, analysis, and reported results in the literature make it challenging to glean systematic relationships between well-being and the brain.

One basic limitation to summarizing extant neuroimaging findings regarding the relationship between well-being and the brain is the inconsistent use of labels for brain areas in the literature. The neuroscience community has begun to try to address difficulties with the nomenclature of neuroanatomy through the development of databases, such as Neurosynth (http://neurosynth.org/) (Yarkoni, Poldrack, Nichols, Van Essen, \& Wager, 2011) and BrainMap (http://www. brainmap.org/) (Fox \& Lancaster, 2002; Laird, Lancaster \& Fox, 2005). These databases consist of published functional and structural neuroimaging experiments with coordinatebased results $(\mathrm{x}, \mathrm{y}, \mathrm{z})$ reported in standard reference spaces (Talairach or MNI space). The software and tools available through these resources allow for the automated extraction of brain coordinates from hundreds of neuroimaging studies (Müller et al., 2017). This approach to collating neuroimaging results provides a standardized classification scheme by which researchers can compare findings across studies. Using such 
resources, scientists can easily conduct meta-analyses of human brain function and structure, identifying and synthesizing patterns in brain activation reported across the literature. Although use of these databases come with various advantages (e.g., faster and less error-prone coordinate extraction), there are various limitations of these resources as well. For example, there are limitations to the search parameters available through these databases, making conducting a comprehensive systematic literature search challenging. In addition, these databases only consist of a subset of the extant neuroimaging literature. In the case of the current review, only two of the included articles (Kong, Hu, Wang, et al., 2015; Lewis et al., 2014) were available through these databases. Therefore, it was not possible to use these tools to synthesize across the literature included in this review. Future efforts should be put toward coding studies for inclusion in these databases, because they provide scientists with a tool to help report neuroimaging findings more consistently.

Another challenge in summarizing published neural correlates of well-being is the methodological variation in neuroimaging research (Carp, 2012; Eklund, Nichols, \& Knutsson, 2016). The emergence of an association between the brain and behavior can be affected by many variables associated with, but not meaningfully related to, the actual brain-behavior relationship. For example, neuroimaging work is particularly susceptible to artifacts caused by respiration, heartbeat, and motion. Many different processing techniques and methods can be used to try to account for such "noise" in the data (e.g., global signal regression); however, different processing techniques and methods in of themselves can also differentially affect observed brain-behavior relationships. At this point in time, understanding neural activity is largely in a period of exploration. At a mechanistic level, our power to predict how the brain is associated with behavior is limited. In the context of most complex behaviors and traits, including well-being, multiple differing relationships might be expected (in possibly positive, negative, or nonlinear directions). Given that there is not a strong basis on which to interpret the direction of the association between well-being and the brain, we can only reasonably assess the absolute relationship between these variables.

In addition to neuroimaging limitations, variability in the definition and measurement of well-being across studies also may have contributed to the widespread findings in the literature. Many terms for well-being are employed in the literature (e.g., psychological well-being, subjective well-being, quality of life, satisfaction, happiness, morale, positive affect, affective balance, perceived well-being, perceived life quality, elation, sense of well-being, subjective welfare). While many of these terms are related, they are not necessarily synonymous. Given the diverse and extensive criteria for wellbeing presented in the literature, it is difficult to determine which among the many descriptions capture the most critical components of well-being. Even a broad definition of wellbeing such as that used in the current review may not capture all facets of well-being.

Regarding the measurement of well-being, all studies in the current review used instruments that have been shown to be both reliable and valid (see Appendix 1). In addition, many measures were validated in multiple cultural populations, suggesting that the instruments account for cultural differences in what constitutes well-being. The measures used also tend to show high convergent validity with other well-being-related measures, suggesting that they all capture similar constructs. Despite these strengths, the inclusion of multiple well-being measures may nonetheless add variability to the results. As outlined in the introduction, the construct of well-being is complex, capturing various elements of positive functioning. While all the measures used in the studies included in the current review tapped into aspects of positive functioning generally (i.e., fell within a broad definition of well-being), the specific components of well-being measured in each instrument differed. Too few studies used the same measure of wellbeing to meaningfully examine the results according to the well-being instrument used. In addition, even in cases where the same well-being measures were used across studies, there were some instances in which scores on the well-being measures were calculated differently across studies, making it difficult to synthesize findings.

Variability in the literature also might be explained in part by methodological limitations in the included studies. While the studies included in the current review were generally methodologically sound, some specific methodological issues should be noted. First, a problem of circular analyses was identified in Luo et al. (2014). Specifically, the authors conducted analyses to explore whether resting-state regional activity was correlated with happiness. To do this, the authors selected brain regions that showed significant group differences between happy and unhappy groups on the same measures of happiness later used in the correlational analyses. This is problematic, as the areas used in the region of interest correlation analyses were not defined independently of the data being examined. Second, multiple studies in the current review either failed to correct for multiple comparisons (Fritz et al., 2016; Waytz et al., 2015; Martikainen et al., 2015) or used a liberal significance threshold (Luo et al., 2016; Hermes et al., 2011), making findings more vulnerable to type I error.

Differences in sample sizes across studies may also contribute to variability in reported results. Studies included in the current review ranged in size from 16 to 299 participants. Small sample sizes are prevalent in neuroimaging work, in large part due to logistical limitations. Research indicates that the median statistical power in the field of neuroscience generally is $21 \%$ (Button et al., 2013). Depending on the effect being investigated, studies with small samples may not have enough power to detect significant effects. Based on the 
literature reviewed, the average relationship between wellbeing and the brain is small (e.g., $\sim r=0.30$ ). Given this generally small effect size, larger samples are needed to detect a significant relationship between well-being and the brain. As a rough guideline based on the studies included in the current review, to detect an effect around $r=0.30$ with $80 \%$ certainty requires at least 85 participants (Fig. 6). By this measure, only 11 of 22 studies included in this review had adequate samples. Two additional studies had nearly adequate samples $(\mathrm{N}=84)$.

Multiple studies in the current review reported no significant relationships between well-being and the brain (Fritz et al., 2016; Martikainen et al., 2015; De Pascalis et al., 2013; Hagemann et al., 1999). These studies ranged in sample size from 16 to 51 participants. Although some studies with similarly low sample sizes reported significant findings, the null results reported in these studies may reflect inadequate power.

Another significant limitation in the literature is the prevalence of nonindependent cohorts across studies. In particular, 7 of 22 studies meeting criteria for the current review were conducted by Kong and colleagues and consisted of groups of highly overlapping participants (as confirmed by the authors). Given that approximately one third of the studies that make up the body of literature examining well-being and the brain come from a single group using highly overlapping samples, it is possible that this set of studies could unduly influence the results of the current review. In addition to the seven studies by Kong and colleagues, two studies in the current review were conducted by Luo and colleagues. Notably, the study conducted by Luo et al. in 2016 included all 50 participants originally included in their 2014 work. This too could potentially unfairly influence the literature.

To briefly quantify if any one group unfairly biased the current review or was an outlier in terms of observed effects, effect sizes were averaged for each group. Given that all of the participants in Luo et al. (2014) were included in the group's later work, only effect sizes from Luo et al. (2016) were included. Averaging across effect sizes resulted in 15 independent effects reported in the literature. Possible publication bias was tested using Egger's Test of the Intercept (Egger, Davey Smith, Schneider, \& Minder, 1997), which provides a statistical test of the relation between sample size and effect size. Results of Egger's test did not show evidence of publication bias $(z=-0.97, p=0.33)$. In addition, three outlier analyses (standardized residuals, leave-one-out analysis, visual inspection of forest plot) were conducted to verify that no one study

\section{approximate correlation power calculation (arctangh transformation)}

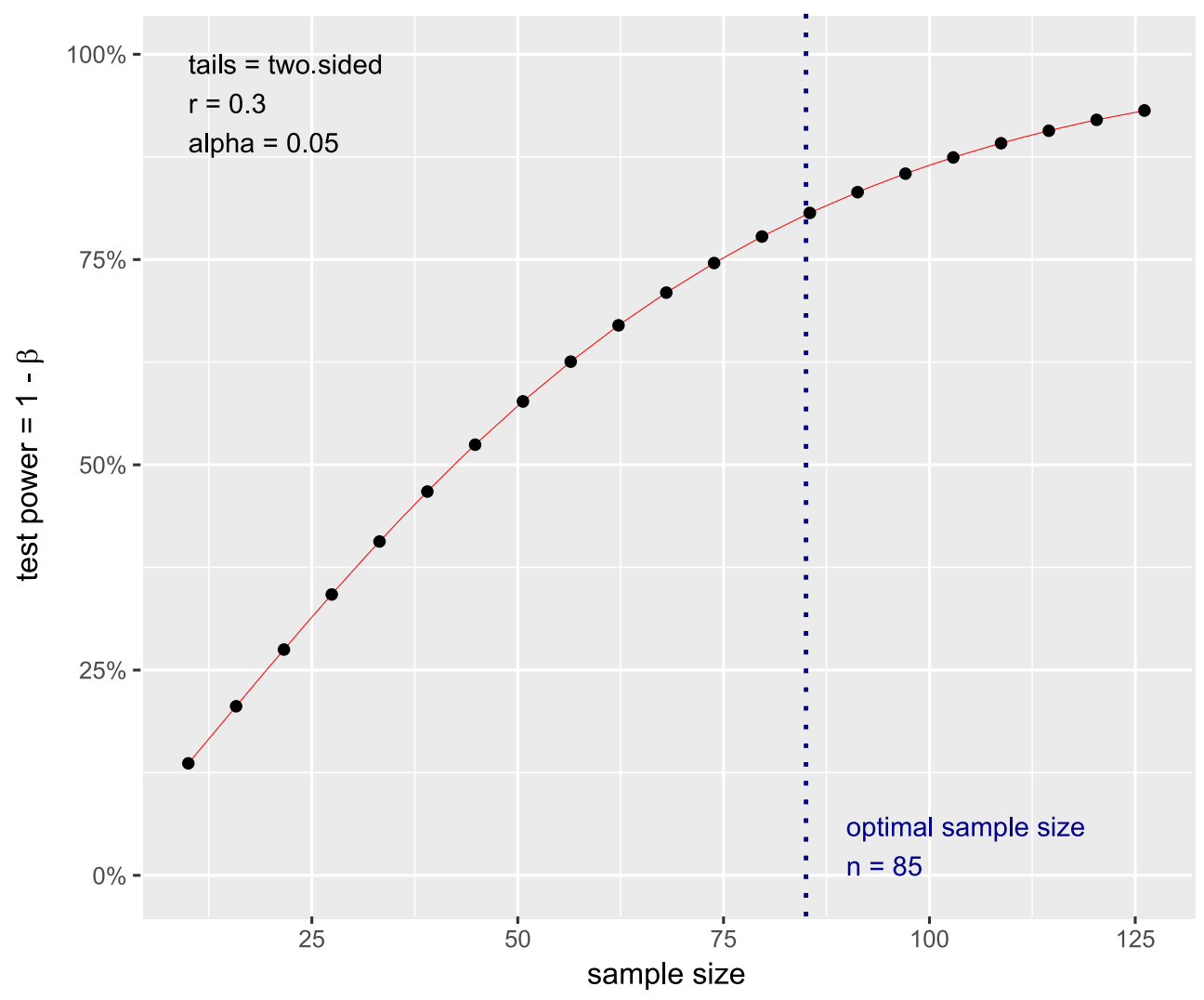

Fig. 6. Power calculation for detecting a significant relationship between well-being and the brain 
was disproportionately affecting the results. No outliers were identified using these methods (see Supplemental Figures 1 and 2).

Given the limitations in the literature, it may be premature to draw conclusions regarding the association between wellbeing and the brain. Although the current work does not allow for definitive conclusions regarding the relationship between well-being and the brain to be drawn, it contributes to the field in multiple, important ways, nonetheless. First, to our knowledge, this is the only systematic synthesis of the extant findings on the neural correlates of well-being. Although the current review highlights the fact that, at this point, our understanding of the relationship between well-being and the brain is limited, largely by methodological problems, it also reveals some noteworthy patterns of association between well-being and the brain that are ripe for further examination.

\section{Future directions}

It is clear that further work needs to be conducted to clarify and confirm the neural correlates of well-being. However, based on the findings from the current review, it also is clear that the field does not simply need more studies exploring this relationship. Rather, a recalibration in the approach to studying the neural correlates of well-being is needed.

Future work should aim to sharpen the focus of the field. A two pronged, hypothesis-driven approach to investigating the neural correlates of well-being may be useful in this endeavor. Given the current findings that well-being is associated with behaviorally well-studied brain systems (e.g., the salience network is implicated in emotion-based tasks), one potential area of future investigation might examine the behavioral correlates of well-being.

At this point in time, well-being is most typically measured using self-report measures. Arguably, there is no one or even set of behaviors that constitute well-being; rather, well-being consists of a constellations of factors. Many studies have examined how well-being is related to other variables, including demographics, mood, personality, emotion, physical health, etc. (Ryan \& Deci, 2001). These studies often are conducted by correlating self-reported measures of well-being with selfreported measures of the variable of interest. Few studies have been conducted examining how behavioral paradigms are related to well-being.

In psychology, the "gold standard" for operationalizing a construct involves identifying a behavior that is reliably related to the given construct or designing a task that captures elements of the construct. Future work examining well-being should investigate how much variance in self-reported wellbeing measures is captured by different behavioral tasks. Established laboratory tasks that may be theoretically linked with well-being, such as, but not limited to, emotion regulation paradigms, social rejection paradigms, and empathy paradigms, might be investigated in future work to examine their relationship to well-being. Having meaningful and consistent behavioral measures of well-being will be important to advancing the study of this complex construct.

A better understanding of the behavioral correlates of wellbeing can also be used to recalibrate the investigation of the neural correlates of well-being. Studies investigating the neural correlates of well-being often take one of two modular approaches. Most studies identify regions of interest for investigation by examining areas of the brain that have been previously linked to constructs related to well-being (e.g., self-efficacy, personality, mood, etc.). Typically, studies use selfreport measures of the constructs related to well-being to identify regions of interest and then examine how self-reported well-being relates to neural structure or function in these brain areas. Less frequently, studies examining the neural correlates of well-being have used task-based approaches. Studies using task-based approaches typically examine the relationship between self-report measures of well-being and neural activation in response to a particular task (Heller et al., 2013).

These approaches limit the inferential power of many of the current studies investigating the relationship between wellbeing and the brain. In particular, these approaches are problematic in that they introduce multiple layers from which researchers must extrapolate an understanding of the relationship between well-being and the brain. Slightly modifying the current approaches to investigating the neural correlates of well-being by using behavioral paradigms related to wellbeing to identify regions of interest could help with the problem of reverse inference. If future work is able to identify meaningful behavioral paradigms that explain a significant amount of variance in self-report measures of well-being, fMRI studies can be designed in which these relevant tasks are used to identify regions of interest. Following the identification of regions of interest using task-based fMRI, these regions can be used as seeds in resting-state analyses to elucidate the relationship between well-being and the brain. The use of converging methods to conduct analyses investigating the neural correlates of well-being may be one way to help hone our current understanding of this complex relationship.

Another possible approach to investigating the neural correlates of well-being is to use a lesion-based method. Although lesion approaches typically use a lesion-deficit model, it is reasonable to apply similar approaches when examining positive functioning (King, Manzel, Bruss \& Tranel, 2017). The same principle (i.e., investigating the relationship between neuroanatomy and some higher order construct) applies regardless of the particular construct being measured. In fact, most of what is known about the functional organization of the human brain is derived from work examining patients with specific neurological conditions (e.g., brain lesions) and observing the behavioral changes associated with localized neuroanatomical damage (Caramazza, 1992). 
Future work should aim to characterize if and how lesions to the ACC, PCC, OFC, STG, and/or thalamus modulate wellbeing. Research suggests that when damaged, the ACC is known to lead to emotional instability, apathy, avolition, and inattention (for review see Szczepanski \& Knight, 2014). How such damage affects well-being should be investigated. In addition, using recently developed lesion network mapping techniques (Fox, 2018), future work might provide evidence that lesions to the ACC, PCC, OFC, STG, and/or thalamus disrupt the salience and/or default mode networks, thereby affecting well-being. Moreover, the lesion network mapping technique can extend our understanding of the neural correlates of well-being by identifying if lesions in other brain areas, which may or may not be associated with changes in well-being, are implicated in either the salience or default mode networks.

As evidenced in this review, research investigating the neural correlates of well-being has not yet been definitive. Future work should focus on transparency and replicability, clearly operationalizing well-being and outlining how neural correlates are measured. Consistency across studies will be crucial to elucidating the neural correlates of well-being. The relationship between well-being and the brain is still in a state of exploration and continued work in this area is critical. Understanding the neural correlates of well-being may help scientists and clinicians develop more targeted strategies and therapies to maximize well-being in various populations. A clearer understanding of the neural correlates of well-being might set the stage for future work investigating how to target well-being in the face of physical or psychological suffering.

Acknowledgments Jennifer Deberg, a health sciences librarian at the University of Iowa with expertise in searches for systematic reviews, helped to develop the search strategies used in the current review.

This work was supported the National Institutes of Health Predoctoral Training Grant (T32-GM108540).

\section{Compliance with ethical standards}

\section{Conflict of interest None}

Open practices statement The data or materials for the review reported are available upon request.

\section{Appendix 1}

Table 3 Included measures of well-being

\begin{tabular}{|c|c|}
\hline Well-being measure & Reference \\
\hline Chinese Happiness Inventory (CHI) & $\begin{array}{l}\text { Lu L, Shih JB (1997a) Personality and happiness: is mental health a mediator? Personality and individual } \\
\text { differences, 22, 249-256. }\end{array}$ \\
\hline $\begin{array}{l}\text { Japanese version of Purpose in Life } \\
\text { Test (PIL) }\end{array}$ & $\begin{array}{l}\text { Sato F et al. (1993) Validity and reliability of Japanese version of PIL (The purpose-in-life test). Iwate Daigaku } \\
\text { Jinbunshakaigakubu Kiyou, 52, 85-97. }\end{array}$ \\
\hline $\begin{array}{l}\text { Japanese version of the Emotional } \\
\text { Intensity Scale }\end{array}$ & $\begin{array}{l}\text { Noguchi M, Sato W, Yoshikawa S (2008) Development of the emotional intensity scale: Japanese version. Taijin } \\
\text { Shakai Shinrigaku Kenkyu, 8, 103-110. }\end{array}$ \\
\hline Japanese version of WHOQOL-26 & Tazaki M., Nakane M (2007) Revised Manual of WHO QOL 26. Tokyo: Kaneko-Syobou. \\
\hline Meaning in Life Questionnaire (MLQ) & $\begin{array}{l}\text { Steger MF, Frazier P, Oishi S, Kaler M (2006) The meaning in life questionnaire: Assessing the presence of and } \\
\text { search for meaning in life. Journal of Counseling Psychology, 53, 80-93. }\end{array}$ \\
\hline $\begin{array}{l}\text { Multidimensional Personality } \\
\text { Questionnaire (MPQ) }\end{array}$ & $\begin{array}{l}\text { Patrick CJ, Curtin JJ, Tellegen A (2002) Development and validation of a brief form of the Multidimensional } \\
\text { Personality Questionnaire. Psychological Assessment,14,150-163. }\end{array}$ \\
\hline $\begin{array}{l}\text { Orientation to Happiness } \\
\text { Questionnaire (OHQ) }\end{array}$ & $\begin{array}{l}\text { Peterson C, Park N, Seligman ME (2005) Orientations to happiness and life satisfaction: the full life versus the } \\
\text { empty life. Journal of Happiness Studies, 6, 25-41. }\end{array}$ \\
\hline $\begin{array}{l}\text { Positive Affect Negative Affect } \\
\text { Schedule (PANAS) }\end{array}$ & $\begin{array}{l}\text { Watson D, Clark LA, Tellegen A (1988) Development and validation of brief measures of positive and negative } \\
\text { affect: the PANAS scales. Journal of Personality and Social Psychology, 54, 1063-1070. }\end{array}$ \\
\hline Rosenberg Self-Esteem Scale (RSES) & Rosenberg M (1965) Society and the Adolescent Self-Image. Princeton, NJ: Princeton University Press. \\
\hline Satisfaction with Life Scales (SWLS) & $\begin{array}{l}\text { Diener E, Emmons RA, Larsen RJ, Griffin S (1985) The Satisfaction with Life Scale. Journal of Personality } \\
\text { Assessment, 49, 71-75. }\end{array}$ \\
\hline $\begin{array}{l}\text { Scales of Psychological Well-being } \\
\quad \text { (SPWB) }\end{array}$ & $\begin{array}{l}\text { Ryff CD (1989) Happiness is everything, or is it? Explorations on the meaning of psychological well-being. } \\
\text { Journal of Personality and Social Psychology, 57, 1069-1081. }\end{array}$ \\
\hline Social Well-Being Scale (SWBS) & Keyes CLM (1998) Social well-being. Social Psychology Quarterly, 61, 121-140. \\
\hline Subjective Happiness Scale (SHS) & $\begin{array}{l}\text { Lyubomirsky S, Lepper H (1999) A measure of subjective happiness: Preliminary reliability and construct } \\
\text { validation. Social Indicators Research, 46, 137-155. }\end{array}$ \\
\hline Short Form-36 (SF-36) & $\begin{array}{l}\text { Ware JE, Sherbourne CD (1992) The MOS 36-item short-form health survey (SF-36). Medical Care, } 30 \text {, } \\
\text { 473-483. [PubMed: 1593914] }\end{array}$ \\
\hline
\end{tabular}




\section{Appendix 2}

Table 4 Exclusion information

\begin{tabular}{ll}
\hline Reason excluded & Number of articles \\
\hline Abstract only & 3 \\
Not able to locate full report & 1 \\
Not experimental & 1 \\
Only one participant & 1 \\
Epilepsy sample & 3 \\
Included participants with mental health problems & 3 \\
Included participants with non-focal brain lesions & 1 \\
Biofeedback intervention & 3 \\
Emotion induction paradigm & 12 \\
Mood induction paradigm & 15 \\
Task-based imaging paradigm only & 37 \\
No validated measure of well-being & 26 \\
No response from author for additional data & 5 \\
Missing data & 9
\end{tabular}

\section{References}

Asterisks (*) indicate studies included in the review.

Allport, G. W. (1961). Pattern and growth in personality. New York: Holt, Rinehart \& Winston.

Andrews-Hanna, J. R. (2012). The brain's default network and its adaptive role in internal mentation. The Neuroscientist, 18(3), 251-270.

Andrews, F. M., \& Withey, S. (1976). Social indicators of well-being: American perceptions of quality of life. The Management Group.

Barrett, L. F., \& Simmons, W. K. (2015). Interoceptive predictions in the brain. Nature Reviews Neuroscience, 16(7), 419.

Berridge, K. C., \& Kringelbach, M. L. (2011). Building a neuroscience of pleasure and well-being. Psychology of Well-Being: Theory, Research and Practice, 1(1), 3.

Bradburn, N. M. (1969). The structure of psychological well-being. Oxford, England: Aldine.

Buckner, R. L., Andrews-Hanna, J. R., \& Schacter, D. L. (2008). The brain's default network. Annals of the New York Academy of Sciences, 1124(1), 1-38.

Bush, G., Luu, P., \& Posner, M. I. (2000). Cognitive and emotional influences in anterior cingulate cortex. Trends in Cognitive Sciences, 4(6), 215-222.

Button, K. S., Ioannidis, J. P., Mokrysz, C., Nosek, B. A., Flint, J., Robinson, E. S., \& Munafò, M. R. (2013). Power failure: Why small sample size undermines the reliability of neuroscience. Nature Reviews Neuroscience, 14(5), 365.

Caramazza, A. (1992). Is cognitive neuropsychology possible? Journal of Cognitive Neuroscience, 4, 80-95.

Carp, J. (2012). On the plurality of (methodological) worlds: Estimating the analytic flexibility of fMRI experiments. Frontiers in Neuroscience, 6, 149.

Chen, X., Yang, R., Kuang, D., Zhang, L., Lv, R., Huang, X., ... Ou, S. (2017). Heart rate variability in patients with major depression disorder during a clinical autonomic test. Psychiatry Research, 256, 207-211.
Cooke, P. J., Melchert, T. P., \& Connor, K. (2016). Measuring well-being: A review of instruments. The Counseling Psychologist, 44(5), 730757.

Craig, A. D. (2002). How do you feel? Interoception: The sense of the physiological condition of the body. Nature Reviews Neuroscience, $3(8), 655$.

Critchley, H. D. (2005). Neural mechanisms of autonomic, affective, and cognitive integration. Journal of Comparative Neurology, 493(1), 154-166.

Davidson, R. J. (2004). Well-being and affective style: Neural substrates and biobehavioural correlates. Philosophical Transactions of the Royal Society B: Biological Sciences, 359(1449), 1395-1411. https://doi.org/10.1098/rstb.2004.1510

*De Pascalis, V., Cozzuto, G., Caprara, G. V., \& Alessandri, G. (2013). Relations among EEG-alpha asymmetry, BIS/BAS, and dispositional optimism. Biological Psychology, 94(1), 198-209. https://doi.org/ 10.1016/j.biopsycho.2013.05.016

Diener, E., Emmons, R. A., Larsen, R. J., \& Griffin, S. (1985). The satisfaction with life scale. Journal of Personality Assessment, 49(1), 71-75.

Disabato, D. J., Goodman, F. R., Kashdan, T. B., Short, J. L., \& Jarden, A. (2016). Different types of well-being? A cross-cultural examination of hedonic and eudaimonic well-being. Psychological Assessment, 28(5), 471

Egger, M., Davey Smith, G., Schneider, M., \& Minder, C. (1997). Bias in meta-analysis detected by a simple, graphical test. The BMJ, 315(7109), 629-634.

Eklund, A., Nichols, T. E., \& Knutsson, H. (2016). Cluster failure: Why fMRI inferences for spatial extent have inflated false-positive rates. Proceedings of the National Academy of Sciences, 113(28), 79007905.

Feinstein, J. S., Khalsa, S. S., Yeh, H., Al Zoubi, O., Arevian, A. C., Wohlrab, C., ... Paulus, M. P. (2018). The elicitation of relaxation and interoceptive awareness using floatation therapy in individuals with high anxiety sensitivity. Biological Psychiatry: Cognitive Neuroscience and Neuroimaging, 3(6), 555-562.

Feinstein, J. S., Khalsa, S. S., Yeh, H. W., Wohlrab, C., Simmons, W. K., Stein, M. B., \& Paulus, M. P. (2018). Examining the short-term anxiolytic and antidepressant effect of Floatation-REST. PloS One, 13(2), e0190292.

Fox, M. D. (2018). Mapping symptoms to brain networks with the human connectome. New England Journal of Medicine, 379(23), 22372245.

Fox, P.T., Lancaster, J.L., 2002. Opinion: Mapping context and content: The BrainMap model. Nature Reviews Neuroscience. 3, 319-321.

*Fritz, N. E., Roy, S., Keller, J., Prince, J., Calabresi, P. A., \& Zackowski, K. M. (2016). Pain, cognition and quality of life associate with structural measures of brain volume loss in multiple sclerosis. NeuroRehabilitation, 39(4), 535-544. https://doi.org/10.3233/ NRE-161384

Greicius, M. D., Krasnow, B., Reiss, A. L., \& Menon, V. (2003). Functional connectivity in the resting brain: A network analysis of the default mode hypothesis. Proceedings of the National Academy of Sciences, 100(1), 253-258.

*Hagemann, D., Naumann, E., Lürken, A., Becker, G., Maier, S., \& Bartussek, D. (1999). EEG asymmetry, dispositional mood and personality. Personality and Individual Differences, 27(3), 541-568.

Hamilton, J. P., Farmer, M., Fogelman, P., \& Gotlib, I. H. (2015). Depressive rumination, the default-mode network, and the dark matter of clinical neuroscience. Biological psychiatry, 78(4), 224-230.

Heller, A. S., van Reekum, C. M., Schaefer, S. M., Lapate, R. C., Radler, B. T., Ryff, C. D., \& Davidson, R. J. (2013). Sustained striatal activity predicts eudaimonic well-being and cortisol output. Psychological Science, 24(11), 2191-2200. https://doi.org/10.1177/ 0956797613490744 
*Hermes, M., Hagemann, D., Naumann, E., \& Walter, C. (2011). Extraversion and its positive emotional core-further evidence from neuroscience. Emotion, 11(2), 367-378. https://doi.org/10.1037/ a0021550

Higgins, J. P. T., \& Green, S. (Eds.). (2011). Cochrane handbook for systematic reviews of interventions cersion 5.1.0 [updated March 2011]. The Cochrane Collaboration. Available from www. handbook.cochrane.org.

Jung, C.G. (1933). Modem man in search of a soul. W. S. Dell, \& C. F. Baynes, Trans. New York: Harcourt, Brace, \& World.

Kahneman, D., Diener, E., \& Schwarz, N. (1999). Well-being: Foundations of hedonic psychology: New York, NY: Russell Sage Foundation.

Kaiser, R. H., Andrews-Hanna, J. R., Wager, T. D., \& Pizzagalli, D. A. (2015). Large-scale network dysfunction in major depressive disorder: A meta-analysis of resting-state functional connectivity. JAMA Psychiatry, 72(6), 603-611.

Kemp, A. H., Quintana, D. S., Gray, M. A., Felmingham, K. L., Brown, K., \& Gatt, J. M. (2010). Impact of depression and antidepressant treatment on heart rate variability: A review and meta-analysis. Biological Psychiatry, 67(11), 1067-1074.

Keyes, C. L., Shmotkin, D., \& Ryff, C. D. (2002). Optimizing well-being: The empirical encounter of two traditions. Journal of Personality and Social Psychology, 82(6), 1007.

King, M. L., Manzel, K., Bruss, J., \& Tranel, D. (2017). Neural correlates of improvements in personality and behavior following a neurological event. Neuropsychologia.

*Kong, F., Ding, K., Yang, Z., Dang, X., Hu, S., Song, Y., \& Liu, J. (2015). Examining gray matter structures associated with individual differences in global life satisfaction in a large sample of young adults. Social Cognitive and Affective Neuroscience, 10(7), 952960. https://doi.org/10.1093/scan/nsu144

*Kong, F., Hu, S., Wang, X., Song, Y., \& Liu, J. (2015). Neural correlates of the happy life: The amplitude of spontaneous low frequency fluctuations predicts subjective well-being. Neuroimage, 107, 136145. https://doi.org/10.1016/j.neuroimage.2014.11.033

*Kong, F., Hu, S., Xue, S., Song, Y., \& Liu, J. (2015). Extraversion mediates the relationship between structural variations in the dorsolateral prefrontal cortex and social well-being. Neuroimage, 105, 269-275. https://doi.org/10.1016/j.neuroimage.2014.10.062

*Kong, F., Liu, L., Wang, X., Hu, S., Song, Y., \& Liu, J. (2015). Different neural pathways linking personality traits and eudaimonic well-being: A resting-state functional magnetic resonance imaging study. Cognitive, Affective, and Behavioral Neuroscience, 15(2), 299-309. https://doi.org/10.3758/s13415-014-0328-1

*Kong, F., Wang, X., Hu, S., \& Liu, J. (2015). Neural correlates of psychological resilience and their relation to life satisfaction in a sample of healthy young adults. Neuroimage, 123, 165-172. https://doi.org/10.1016/j.neuroimage.2015.08.020

*Kong, F., Wang, X., Song, Y., \& Liu, J. (2016). Brain regions involved in dispositional mindfulness during resting state and their relation with well-being. Social Neuroscience, 11(4), 331-343. https://doi. org/10.1080/17470919.2015.1092469

*Kong, F., Xue, S., \& Wang, X. (2016). Amplitude of low frequency fluctuations during resting state predicts social well-being. Biological Psychology, 118, 161-168. https://doi.org/10.1016/j. biopsycho.2016.05.012

Laird, A.R., Lancaster, J.L., Fox, P.T., 2005. BrainMap: The social evolution of a human brain mapping database. Neuroinformatics, 3, 6578.

*Lewis, G. J., Kanai, R., Rees, G., \& Bates, T. C. (2014). Neural correlates of the 'good life': Eudaimonic well-being is associated with insular cortex volume. Social Cognitive and Affective neuroscience, 9(5), 615-618.
Lucas, R. E., Diener, E., \& Suh, E. (1996). Discriminant validity of wellbeing measures. Journal of Personality and Social Psychology, $71(3), 616$.

*Luo, Y., Huang, X., Yang, Z., Li, B., Liu, J., \& Wei, D. (2014). Regional homogeneity of intrinsic brain activity in happy and unhappy individuals. PloS One, 9(1), e85181. https://doi.org/10.1371/journal. pone.0085181

*Luo, Y., Kong, F., Qi, S., You, X., \& Huang, X. (2016). Resting-state functional connectivity of the default mode network associated with happiness. Social Cognitive and Affective Neuroscience, 11(3), 516524. https://doi.org/10.1093/scan/nsv132

Lyubomirsky, S., Sheldon, K. M., \& Schkade, D. (2005). Pursuing happiness: The architecture of sustainable change. Review of General Psychology, 9(2), 111-131. https://doi.org/10.1037/1089-2680.9.2. 111

Machado, L., \& Cantilino, A. (2017). Neural correlates of wellbeing scales: Preliminary data. Australian and New Zealand Journal of Psychiatry, 51(9), 946-946. https://doi.org/10.1177/ 0004867417698229

*Martikainen, I. K., Nuechterlein, E. B., Pecina, M., Love, T. M., Cummiford, C. M., Green, C. R., . . Zubieta, J. K. (2015). Chronic back pain is associated with alterations in dopamine neurotransmission in the ventral striatum. Journal of Neuroscience, 35(27), 9957-9965. https://doi.org/10.1523/jneurosci.4605-14.2015

Maslow, A. H. (1968). Toward a psychology of being (2nd ed.). New York: Van Nostrand.

*Matsunaga, M., Kawamichi, H., Koike, T., Yoshihara, K., Yoshida, Y., Takahashi, H. K., . . . Sadato, N. (2016). Structural and functional associations of the rostral anterior cingulate cortex with subjective happiness. Neuroimage, 134, 132-141. https://doi.org/10.1016/j. neuroimage.2016.04.020

Mulders, P. C., van Eijndhoven, P. F., Schene, A. H., Beckmann, C. F., \& Tendolkar, I. (2015). Resting-state functional connectivity in major depressive disorder: A review. Neuroscience \& Biobehavioral Reviews, 56, 330-344.

Müller, V. I., Cieslik, E. C., Laird, A. R., Fox, P. T., Radua, J., MataixCols, D., . . . Turkeltaub, P. E. (2017). Ten simple rules for neuroimaging meta-analysis. Neuroscience \& Biobehavioral Reviews.

Paus, T. (2001). Primate anterior cingulate cortex: Where motor control, drive and cognition interface. Nature Reviews Neuroscience, 2(6), 417.

Peterson, C., Park, N., \& Seligman, M. E. (2005). Orientations to happiness and life satisfaction: The full life versus the empty life. Journal of Happiness Studies, 6(1), 25-41.

Peyron, R., Laurent, B., \& Garcia-Larrea, L. (2000). Functional imaging of brain responses to pain. A review and meta-analysis (2000). Neurophysiologie Clinique/Clinical Neurophysiology, 30(5), 263288.

Quoidbach, J., Berry, E. V., Hansenne, M., \& Mikolajczak, M. (2010). Positive emotion regulation and well-being: Comparing the impact of eight savoring and dampening strategies. Personality and Individual Differences, 49(5), 368-373.

Rogers, C. R. (1961). On becoming a person. Boston: Houghton Muffin.

Ross, W. D. (1925). The oxford translation of Aristotle, Vol. IX: The nichomachean ethics.

Ryan, R. M., \& Deci, E. L. (2001). On happiness and human potentials: A review of research on hedonic and eudaimonic well-being. Annual Review of Psychology, 52(1), 141-166.

Ryff, C. D. (1989). Happiness is everything, or is it? Explorations on the meaning of psychological well-being. Journal of Personality and Social Psychology, 57(6), 1069.

*Sato, W., Kochiyama, T., Uono, S., Kubota, Y., Sawada, R., Yoshimura, S., \& Toichi, M. (2015). The structural neural substrate of subjective happiness. Scientific Reports, 5, 16891. https://doi.org/10.1038/ srep16891 
Schaffer, C. E., Davidson, R. J., \& Saron, C. (1983). Frontal and parietal electroencephalogram asymmetry in depressed and nondepressed subjects. Biological Psychiatry.

Seeley, W. W., Menon, V., Schatzberg, A. F., Keller, J., Glover, G. H., Kenna, H., ... Greicius, M. D. (2007). Dissociable intrinsic connectivity networks for salience processing and executive control. Journal of Neuroscience, 27(9), 2349-2356.

Seligman, M. E. P., \& Csikszentmihalyi, M. (2014). Positive psychology: An introduction. Flow and the foundations of positive psychology: The collected works of Mihaly Csikszentmihalyi (pp. 279-298). Dordrecht: Springer.

Szczepanski, S. M., \& Knight, R. T. (2014). Insights into human behavior from lesions to the prefrontal cortex. Neuron, 83(5), 1002-1018.

*Takeuchi, H., Taki, Y., Nouchi, R., Hashizume, H., Sassa, Y., Sekiguchi, A., . . Kawashima, R. (2014). Anatomical correlates of quality of life: Evidence from voxel-based morphometry. Human Brain Mapping, 35(5), 1834-1846.https://doi.org/10.1002/hbm.22294

*Tomarken, A. J., Davidson, R. J., Wheeler, R. E., \& Doss, R. C. (1992). Individual differences in anterior brain asymmetry and fundamental dimensions of emotion. Journal of Personality and Social Psychology, 62(4), 676-687.

*Urry, H. L., Nitschke, J. B., Dolski, I., Jackson, D. C., Dalton, K. M., Mueller, C. J., . . Davidson, R. J. (2004). Making a life worth living: Neural correlates of well-being. Psychological Science, 15(6), 367372. https://doi.org/10.1111/j.0956-7976.2004.00686.x
*Volkow, N. D., Tomasi, D., Wang, G. J., Fowler, J. S., Telang, F., Goldstein, R. Z., . . . Alexoff, D. (2011). Positive emotionality is associated with baseline metabolism in orbitofrontal cortex and in regions of the default network. Molecular Psychiatry, 16(8), 818825. https://doi.org/10.1038/mp.2011.30

Waterman, A. S. (1984). The psychology of individualism. Westport, CT: Praeger Publishers.

Watson, D., Clark, L. A., \& Tellegen, A. (1988). Development and validation of brief measures of positive and negative affect: The PANAS scales. Journal of Personality and Social Psychology, 54(6), 1063.

*Waytz, A., Hershfield, H. E., \& Tamir, D. I. (2015). Mental simulation and meaning in life. Journal of Personality and Social Psychology, 108(2), 336-355. https://doi.org/10.1037/a0038322

Yarkoni, T., Poldrack, R.A., Nichols, T.E., Van Essen, D.C., Wager, T.D., 2011. Large-scale automated synthesis of human functional neuroimaging data. Nature Methods, 8, 665-670.

Publisher's note Springer Nature remains neutral with regard to jurisdictional claims in published maps and institutional affiliations. 